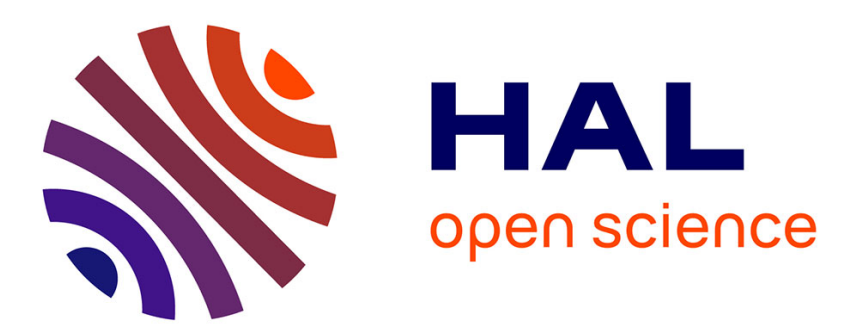

\title{
Estabilização quadrática por realimentação de saída de sistemas Lur'e a tempo contínuo via LMIs
}

Ariádne L J Bertolin, Ricardo C L F Oliveira, Giorgio Valmorbida, Pedro L D

Peres

\section{- To cite this version:}

Ariádne L J Bertolin, Ricardo C L F Oliveira, Giorgio Valmorbida, Pedro L D Peres. Estabilização quadrática por realimentação de saída de sistemas Lur'e a tempo contínuo via LMIs. Congresso Brasileiro de Automática - 2020, Nov 2020, Porto Alegre, Brazil. 10.48011/asba.v2i1.1437 . hal03151866

\section{HAL Id: hal-03151866 \\ https://hal.inria.fr/hal-03151866}

Submitted on 25 Feb 2021

HAL is a multi-disciplinary open access archive for the deposit and dissemination of scientific research documents, whether they are published or not. The documents may come from teaching and research institutions in France or abroad, or from public or private research centers.
L'archive ouverte pluridisciplinaire HAL, est destinée au dépôt et à la diffusion de documents scientifiques de niveau recherche, publiés ou non, émanant des établissements d'enseignement et de recherche français ou étrangers, des laboratoires publics ou privés. 


\title{
Estabilização Quadrática por Realimentação de Saída de Sistemas Lur'e a Tempo Contínuo via LMIs *
}

\author{
Ariádne L. J. Bertolin* Ricardo C. L. F. Oliveira* \\ Giorgio Valmorbida ** Pedro L. D. Peres* \\ * Faculdade de Engenharia Elétrica e de Computação, \\ Universidade Estadual de Campinas - UNICAMP, 13083-852, Campinas, SP, \\ Brasil. (e-mail:\{ariberto,ricfow,peres\}@dt.fee.unicamp.br) \\ ** Laboratoire des Signaux et Systèmes, CentraleSupélec, CNRS, \\ University of Paris-Sud, Université Paris-Saclay, Gif-sur-Yvette 91192, \\ França. (e-mail: giorgio.valmorbida@l2s.centralesupelec.fr)
}

\begin{abstract}
This work investigates the problem of stabilization of continuous-time Lur'e systems with sector bounded nonlinearity. The proposed control law feedbacks both the output and the nonlinearity of the system. The conditions, formulated in terms of linear matrix inequalities, are based on a quadratic Lyapunov function and solved by means of an iterative algorithm. As main novelty, the gains of the controller appear linearly and are treated as decision variables of the optimization problem. Therefore, the presented method can indistinctly handle state and output-feedback, as well as magnitude limitations or structural constraints (such as decentralization) on the gains. Numerical examples illustrate the benefits of the proposed approach, which can be computationally more effective and less conservative when compared to other existing techniques in particular contexts, such as decentralized state-feedback control.

Resumo: Este trabalho investiga o problema de estabilização de sistemas Lur'e contínuos no tempo com não linearidade limitada em setor. A lei de controle proposta é constituída pela realimentação tanto da saída quanto da não-linearidade do sistema. As condições de síntese, formuladas em termos de desigualdades matriciais lineares e baseadas em uma função de Lyapunov quadrática, são resolvidas por meio de um algoritmo iterativo. Como novidade, os ganhos de controle aparecem de forma linear e são tratados como variáveis de decisão do problema de otimização. Portanto, o método apresentado pode lidar com realimentação de estados e de saída indistintamente, além de possibilitar a imposição de limitações de magnitude ou restrições estruturais (como descentralização) sobre os ganhos. Exemplos numéricos ilustram os benefícios da abordagem proposta, que pode ser computacionalmente mais eficaz e menos conservadora quando comparada a outras técnicas existentes em contextos particulares, como a realimentação de estados descentralizada.
\end{abstract}

Keywords: Continuous-time Lur'e systems; output-feedback; quadratic stability; sector bounded non-linearity; linear matrix inequality.

Palavras-chaves: Sistemas Lur'e contínuos no tempo; realimentação de saída; estabilidade quadrática; não linearidade limitada por setor; desigualdade matricial linear.

\section{INTRODUÇÃO}

Os chamados sistemas Lur'e, isto é, sistemas que podem ser descritos por uma planta linear invariante no tempo $\mathscr{G}$ realimentada por uma função não-linear $\phi$ (como ilustra a Fig. 1) satisfazendo certas condições (relacionadas a limitações de setor ou inclinação), representam uma grande família de problemas de engenharia (Khalil, 2002). O problema de análise de estabilidade dos sistemas Lur'e, contínuos ou discretos no tempo, é estudado utilizando uma abordagem temporal com a busca de funções de Lyapunov (Gonzaga et al., 2012; Ahmad et al., 2013; Park, 1997; Park et al., 2019; Kapila and Haddad,

\footnotetext{
* Apoiado pelas agências brasileiras: CNPq, Coordenação de Aperfeiçoamento de Pessoal de Nível Superior - Brasil (CAPES) - Código de Financiamento 001 e Fundação de Amparo à Pesquisa do Estado de São Paulo FAPESP (processo 2019/10947-1).
}

1996; Montagner et al., 2007; Yang et al., 2007; Kalman, 1963; Valmorbida et al., 2019), ou então utilizando multiplicadores de Zames-Falb (Ahmad and Carrasco, 2016; Carrasco et al., 2016; Haddad and Bernstein, 1994, 1993). Nas duas metodologias, a busca por parâmetros das funções de Lyapunov candidatas, ou pelos multiplicadores, pode ser formulada em termos de problemas convexos de otimização, sendo, na maior parte dos casos, problemas de programação semidefinida descritos por desigualdades matriciais lineares (em inglês, Linear Matrix Inequalities - LMIs) (Boyd et al., 1994).

Extensões das condições de análise para tratar o problema de síntese de leis de controle foram foco da atenção de diversos pesquisadores, considerando tanto estabilidade global quanto local (Tarbouriech et al., 2011; Hu et al., 2006). No entanto, com poucas exceções (Lee and Park, 2008; Louis et al., 2015), 
as condições propostas não se baseiam nas funções de Lyapunov mais complexas, restringindo-se a funções quadráticas, quase sempre associadas a uma realimentação linear dos estados. Por exemplo, Castelan et al. (2008) estudam sistemas a tempo contínuo contendo dois tipos de não-linearidades: saturação e limitação de setor. Como lei de controle, propõem uma estratégia de síntese de ganhos de realimentação de estado e das não-linearidades baseada na estabilidade quadrática.

O objetivo deste trabalho é propor leis de controle do tipo realimentação de saída para sistemas Lur'e contínuos e invariantes no tempo com não linearidades limitadas em setor. A lei de controle é composta por ganhos de realimentação estáticos proporcionais à saída medida e ao valor da não linearidade. Utilizando uma função quadrática de Lyapunov, condições suficientes para o projeto dos ganhos estabilizantes são formuladas em termos de LMIs. Como novidade, os ganhos de controle são diretamente variáveis do problema, facilitando a imposição de estruturas particulares, como por exemplo, a descentralizada, sem conservadorismo adicional. Graças a um procedimento de relaxação adequado, as condições de síntese são resolvidas por meio de um algoritmo iterativo. Exemplos ilustram o desempenho do método proposto quando comparado com outras técnicas da literatura.

\section{Notação}

Para uma matriz simétrica $X>0(X<0)$ significa que $X$ é definida positiva (negativa). Para as matrizes ou vetores $\left({ }^{\top}\right)$ indica o transposto, $\operatorname{He}(X)=X+X^{\top}$ e $\operatorname{diag}\left(X_{1}, \ldots, X_{n}\right)$ representa um matriz bloco diagonal formada pelas matrizes quadradas (ou elementos) $X_{1}, \ldots, X_{n}$. O símbolo $\star$ representa um termo induzido por simetria em matrizes quadradas formadas por blocos e o símbolo $\circ$ representa o produto de Hadamard definido como $(X \circ Y)_{i, j}=X_{i, j} Y_{i, j}$. O sinal $(X)$ é o operador sinal e $\lfloor X\rfloor$ é operador piso de cada componente do vetor $X$. As matrizes identidade e nula são denotadas, respectivamente, por $I$ e 0 . Ao longo do texto as dimensões das matrizes podem ser omitidas por simplicidade (sendo inferidas a partir do contexto).

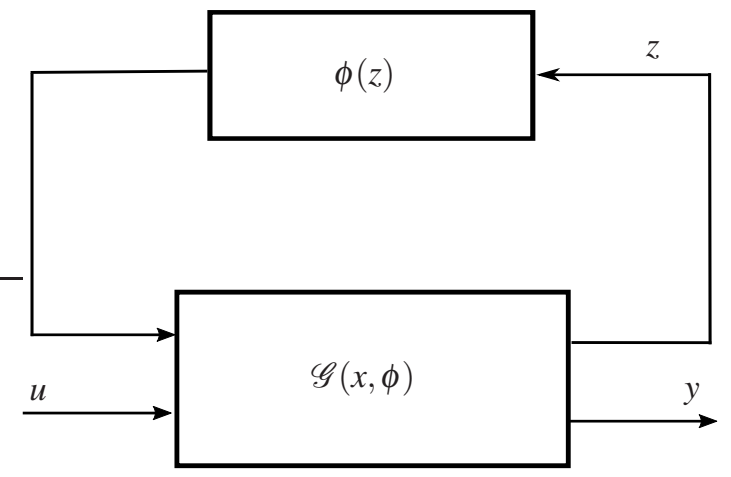

Fig. 1. Diagrama de blocos de um sistema Lur'e em que $\mathscr{G}(x, \phi)$ é um sistema linear invariante no tempo e $\phi(z)$ é uma função não linear limitada em setor.

\section{PRELIMINARES}

Considere o sistema Lur'e a tempo contínuo dado por

$$
\begin{aligned}
\dot{x}(t) & =A x(t)+B_{\phi} \phi(z(t))+B_{u} u(t) \\
z(t) & =C_{z} x(t)+D_{z} \phi(z(t)) \\
y(t) & =C_{y} x(t) .
\end{aligned}
$$

em que $x \in \mathbb{R}^{n_{x}}$ é o vetor de estados, $u \in \mathbb{R}^{n_{u}}$ a entrada de controle, $y \in \mathbb{R}^{n_{y}}$ a saída medida e $z \in \mathbb{R}^{n_{z}}$ é uma saída do bloco linear que passa pela não linearidade. As matrizes do sistema são $A \in \mathbb{R}^{n_{x} \times n_{x}}, B_{\phi} \in \mathbb{R}^{n_{x} \times n_{z}}, B_{u} \in \mathbb{R}^{n_{x} \times n_{u}}, C_{y} \in \mathbb{R}^{n_{y} \times n_{x}}$, $C_{z} \in \mathbb{R}^{n_{z} \times n_{x}}$ e $D_{z} \in \mathbb{R}^{n_{z} \times n_{z}}$.

A não-linearidade $\phi: \mathbb{R}^{n_{z}} \rightarrow \mathbb{R}^{n_{z}}$ é invariante no tempo, descentralizada, i.e. $\phi(z)=\left[\phi_{1}\left(z_{1}\right) \cdots \phi_{n_{z}}\left(z_{n_{z}}\right)\right]^{\top}$, e satisfaz a seguinte hipótese (Khalil, 2002).

Hipótese 1. A não-linearidade $\phi$ verifica

$$
\phi(z)^{\top}[\phi(z)-\Omega z] \leq 0
$$

para todo $z \in \mathbb{R}^{n_{z}}$, sendo $\Omega=\operatorname{diag}\left(\Omega_{1}, \ldots, \Omega_{n_{z}}\right)>0$ uma matriz dada. Além disso, $\phi(0)=0_{n_{z}}$.

A Hipótese 1 corresponde à não linearidade $\phi(z)$ ser limitada em setor, como ilustra a Fig. 2.

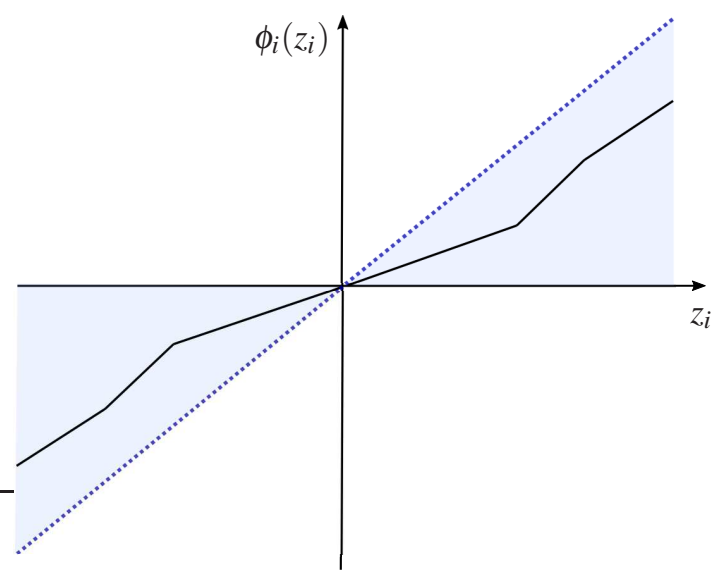

Fig. 2. Não-linearidade $\phi_{i}$ limitada pelo setor $\Omega_{i}$ (azul).

Assume-se que o sistema (1) é bem posto, ou seja, possui um bom comportamento algébrico da realimentação não-linear, propriedade que pode ser garantida mediante a seguinte hipótese.

Hipótese 2.

$$
\operatorname{He}\left(I-\Omega D_{z}\right)>0 .
$$

O problema a ser investigado neste artigo é o projeto da lei de controle dada por

$$
u=K y+L \phi(z) .
$$

em que $K \in \mathbb{R}^{n_{u} \times n_{y}}$ e $L \in \mathbb{R}^{n_{u} \times n_{z}}$ são os ganhos de realimentação associados à saída $y$ e à não linearidade $\phi(z)$, respectivamente.

O lema apresentado a seguir é útil para provar os principais resultados propostos neste trabalho (de Oliveira and Skelton, 2001).

Lema 1. (Lema de Finsler). Considere as matrizes $\mathscr{Q} \in \mathbb{R}^{\ell \times \ell} \mathrm{e}$ $\mathscr{B} \in \mathbb{R}^{m \times \ell}$, com posto $\left.\mathscr{B}\right)<\ell$ e $\mathscr{B} \perp$ representando uma base para o espaço nulo de $\mathscr{B}$, ou seja, $\mathscr{B} \mathscr{B} \perp=0$. As seguintes condições são equivalentes:
i) $\xi^{\top} \mathscr{Q} \xi<0, \forall \xi \in \mathbb{R}^{\ell}, \xi \neq 0, \mathscr{B} \xi=0$;
ii) $\mathscr{B}_{\perp}^{\top} \mathscr{Q} \mathscr{B}_{\perp}<0$;
iii) $\exists \mu \in \mathbb{R}$ tal que $\mathscr{Q}-\mu \mathscr{B}^{\top} \mathscr{B}<0$;
iv) $\exists \mathscr{X} \in \mathbb{R}^{\ell \times m}$ tal que $\mathscr{Q}+\mathscr{X} \mathscr{B}+\mathscr{B}^{\top} \mathscr{X}^{\top}<0$.

\section{RESULTADOS PRINCIPAIS}

Teorema 1. Sejam $\bar{Y}_{i} \in \mathbb{R}^{n_{x} \times n_{x}}, i=1,2,4$ e $\bar{Y}_{3} \in \mathbb{R}^{n_{z} \times n_{x}}$ matrizes dadas. Se existirem matrizes $K \in \mathbb{R}^{n_{u} \times n_{y}}, L \in \mathbb{R}^{n_{u} \times n_{z}}$, 
$Y_{i} \in \mathbb{R}^{n_{x} \times n_{x}}, i=1,2,4, Y_{3} \in \mathbb{R}^{n_{x} \times n_{z}}$ e matrizes definidas positivas $P=P^{\top} \in \mathbb{R}^{n_{x} \times n_{x}}$ e $T \in \mathbb{R}^{n_{z} \times n_{z}}$ diagonal, tais que

$$
\begin{gathered}
{\left[\begin{array}{cccc}
0 & P & C_{z}^{\top} \Omega T & A_{c l}^{T} \\
P & 0 & 0 & -I \\
T \Omega C_{z} & 0 & \operatorname{He}\left(T \Omega D_{z}-T\right) & B_{c l}^{T} \\
A_{c l} & -I & B_{c l} & 0
\end{array}\right]} \\
+\operatorname{He}(\underbrace{\left[\begin{array}{c}
\bar{Y}_{1} \\
\bar{Y}_{2} \\
\bar{Y}_{3} \\
\bar{Y}_{4}
\end{array}\right]}_{\bar{Y}}\left[\begin{array}{llll}
Y_{1} & Y_{2} & Y_{3} & Y_{4}
\end{array}\right])<0
\end{gathered}
$$

é verificada, com

$$
A_{c l}=A+B_{u} K C_{y}, \quad B_{c l}=B_{\phi}+B_{u} L,
$$

então o sistema Lur'e (1) sob as Hipóteses 1 e 2 é globalmente assintoticamente estável em malha fechada com a lei de controle (4).

Prova: Se a desigualdade (5) é verificada, então $\mathrm{He}\left(\bar{Y}_{4} Y_{4}\right)<$ 0 e, portanto, tanto $\bar{Y}_{4}$ como $Y_{4}$ têm posto completo. Dessa maneira, observa-se que a condição (5) pode ser caracterizada como iv) do Lema de Finsler, com $\mathscr{B}=Y$, e as colunas da matriz

$$
\mathscr{B}_{\perp}=\left[\begin{array}{ccc}
I & 0 & 0 \\
0 & I & 0 \\
0 & 0 & I \\
-Y_{4}^{-1} Y_{1} & -Y_{4}^{-1} Y_{2} & -Y_{4}^{-1} Y_{3}
\end{array}\right]
$$

formam uma base para o espaço nulo de $Y$, ou seja, $Y \mathscr{B}_{\perp}=0$. Pelo lema, (5) é equivalente a $\mathscr{B}_{\perp}^{\top} \mathscr{Q} \mathscr{B}_{\perp}<0$, que pode ser reescrito como

$$
\begin{array}{r}
{\left[\begin{array}{clc}
0 & P & C_{z}^{\top} \Omega T \\
P & 0 & 0 \\
T \Omega C_{z} & 0 & \mathrm{He}\left(T \Omega D_{z}-T\right)
\end{array}\right]} \\
\left.+\operatorname{He}\left(\begin{array}{c}
\left(Y_{4}^{-1} Y_{1}\right)^{\top} \\
\left(Y_{4}^{-1} Y_{2}\right)^{\top} \\
\left(Y_{4}^{-1} Y_{3}\right)^{\top}
\end{array}\right]\left[A_{c l}-I B_{c l}\right]\right)<0 .
\end{array}
$$

Novamente, olhando a desigualdade acima como $i v$ ) do Lema de Finsler e computando uma base para o espaço nulo de $\left[A_{c l}-I B_{c l}\right]$, tem-se

$$
\left[\begin{array}{cc}
I & 0 \\
A_{c l} & B_{c l} \\
0 & I
\end{array}\right]^{\top}\left[\begin{array}{ccc}
0 & P & C_{z}^{\top} \Omega T \\
P & 0 & 0 \\
T \Omega C_{z} & 0 & \operatorname{He}\left(T \Omega D_{z}-T\right)
\end{array}\right]\left[\begin{array}{cc}
I & 0 \\
A_{c l} & B_{c l} \\
0 & I
\end{array}\right]<0
$$

ou

$$
\left[\begin{array}{cc}
A_{c l}^{\top} P+P A_{c l} & P B_{c l}+C_{z}^{\top} \Omega T \\
\star & \mathrm{He}\left(T \Omega D_{z}-T\right)
\end{array}\right]<0 .
$$

Multiplicando (6) pela esquerda por $\left[x^{\top} \phi(z)^{\top}\right]$ e pela direita pelo transposto, tem-se

$$
\begin{aligned}
& x^{\top} A_{c l}^{\top} P x+x^{\top} P A_{c l} x+\phi(z)^{\top} T \Omega C_{z} x+\phi(z)^{\top} B_{c l}^{\top} P x \\
& +x^{\top} P B_{c l} \phi(z)+x^{\top} C_{z}^{\top} \Omega T \phi(z)+\phi(z)^{\top} T \Omega D_{z} \phi(z) \\
& +\phi(z)^{\top} D_{z}^{\top} \Omega T \phi(z)-2 \phi(z)^{\top} T \phi(z)<0,
\end{aligned}
$$

que corresponde a

$$
\dot{V}(x)-2 \phi(z)^{\top} T[\phi(z)-\Omega z]<0,
$$

quando $V(x)=x^{\top} P x$ é usada como uma função de Lyapunov quadrática para o sistema (1). Sob a Hipótese 1, o último termo do lado esquerdo da desigualdade acima é sempre positivo para qualquer matriz diagonal $T>0$. Portanto, o sistema (1) em malha fechada é globalmente assintoticamente estável.

A desigualdade apresentada em (5) é uma LMI pois $\bar{Y}_{i}, i=$ $1, \ldots, 4$, são matrizes dadas. Como consequência, é esperado que os resultados obtidos por meio dessa condição suficiente sejam conservadores, devido à falta de uma regra geral para escolher matrizes adequadas $\bar{Y}_{i}, i=1, \ldots, 4$. Uma alternativa para reduzir o conservadorismo, baseada na técnica proposta em Felipe et al. (2016), é introduzir relaxações, como apresentado no próximo teorema.

Teorema 2. Seja

$$
\bar{Y}^{\top}=\left[\begin{array}{llll}
I & I & 0 & -I
\end{array}\right], \quad A_{c l}=A+B_{u} K C_{y}-r I,
$$

em que $r$ é um escalar. Então, as condições do Teorema 1 sempre fornecem soluções factíveis para um valor suficientemente grande de $r>0$.

Prova: Considere a expressão (5) para $A_{c l}$ e $\bar{Y}$ dadas em (7), e adote $Y_{1}=Y_{2}=-Y_{4}=-(r / 2) I, Y_{3}=0$ e $K=L=0$ na desigualdade (5). Então

$$
\begin{gathered}
{\left[\begin{array}{cccc}
0 & P & C_{z}^{\top} \Omega T & A^{\top} \\
P & 0 & 0 & -I \\
T \Omega C_{z} & 0 & H e\left(T \Omega D_{z}-T\right) & B^{\top} \\
A & -I & B & 0
\end{array}\right]} \\
\quad-\frac{r}{2} \operatorname{He}\left(\left[\begin{array}{c}
I \\
I \\
0 \\
-I
\end{array}\right]\left[\begin{array}{llll}
I & I & 0 & -I
\end{array}\right]\right)<0,
\end{gathered}
$$

Escolhendo $P=r I, r>0$, tem-se

$$
\left[\begin{array}{cccc}
-r I & 0 & C_{z}^{\top} \Omega T & A \\
\star & -r I & 0 & (r-1) I \\
\star & \star & \operatorname{He}\left(T \Omega D_{z}-T\right) & B_{\phi}^{\top} \\
\star & \star & \star & -r I
\end{array}\right]<0
$$

ou, de forma equivalente (por complemento de Schur),

$$
r\left[\begin{array}{cc}
\operatorname{He}\left(T-T \Omega D_{z}\right) & -B_{\phi}^{\top} \\
\star & 2 I
\end{array}\right]-\left[\begin{array}{cc}
T \Omega C_{z} C_{z}^{\top} \Omega T & T \Omega C_{z} A^{\top} \\
\star & A A^{\top}+I
\end{array}\right]>0 .
$$

Observa-se que a matriz multiplicada por $r$ pode ser feita definida positiva, pois por complemento de Schur tem-se

$$
2 \operatorname{He}\left(T\left(I-\Omega D_{z}\right)\right)>B_{\phi}^{\top} B_{\phi},
$$

que sempre é verificada considerando a Hipótese 2 e $T=$ $\tau I>0$ com uma escolha adequada para $\tau>0$. Dessa forma, a condição (9) é sempre satisfeita para $r>0$ suficientemente grande.

De acordo com o resultado acima, com as escolhas dadas no Teorema 2, as condições do Teorema 1 sempre produzem solução factível para um $r>0$ suficientemente grande. Uma propriedade importante do fator de relaxação $r$ é que o mesmo aparece de forma afim nas condições do Teorema 1, podendo ser utilizado como função objetivo em um problema de otimização.

Note que a estabilidade da malha fechada exige que as condições sejam satisfeitas com $r=0$ (ou seja, quando as matrizes da malha fechada modificadas fornecidas em (7) se tornam iguais às matrizes originais do sistema). Em vista disso, o próximo teorema fornece um resultado importante para a implementação de um procedimento iterativo baseado nas condições do Teorema 1. 
Teorema 3. Se as condições do Teorema $1 \operatorname{com} A_{c l}$ dada em (7) são factíveis para algum $\hat{r}$, a condição de estabilidade (6) continua factível para qualquer $r>\hat{r}$.

Prova: Considere que as condições do Teorema 1 são factíveis para um dado $\hat{r}$. Então, a condição (6), com $A_{c l}$ dada em (7), é verificada. Reescrevendo a desigualdade, tem-se

$$
\begin{aligned}
& {\left[\begin{array}{cc}
\left(A+B_{u} K C_{y}\right)^{\top} P+P\left(A+B_{u} K C_{y}\right) & P B_{c l}+C_{z}^{\top} \Omega T \\
\star & \operatorname{He}\left(T \Omega D_{z}-T\right)
\end{array}\right]} \\
& -\hat{r}\left[\begin{array}{cc}
2 P & 0 \\
0 & 0
\end{array}\right]<0 \text {. }
\end{aligned}
$$

Considerando $r=\hat{r}+\varepsilon$, a condição acima é satisfeita $\forall \varepsilon>0$ e, portanto, se mantém factível para qualquer $r>\hat{r}$.

A importância do Teorema 3 é a garantia de que se o Teorema 1 for testado com $A_{c l}$ dada em (7), isto é, com condições relaxadas, qualquer solução associada a um $r$ não positivo assegura a estabilidade do sistema em malha fechada, pois a condição de estabilidade (6) permanece factível para qualquer $r$ maior. Em particular, para $r=0$, que recupera o sistema original.

O Algoritmo 1 apresenta o procedimento iterativo proposto neste trabalho para a estabilização do sistema (1) por meio da lei de controle (4). Como parâmetros de entrada, têm-se $i t_{\text {max }}$, o número máximo de iterações, e $\bar{Y}$, uma condição inicial (por exemplo, a apresentada no Teorema 2). O índice $(\cdot)_{k}$ representa o valor da variável na iteração $k$. Os resultados dos teoremas 2 e 3 garantem que o procedimento fornece uma solução factível com um valor finito para $r$ na primeira iteração, e também que, se uma solução com $r \leq 0$ for obtida, a condição (6) permanece válida com $r=0$.

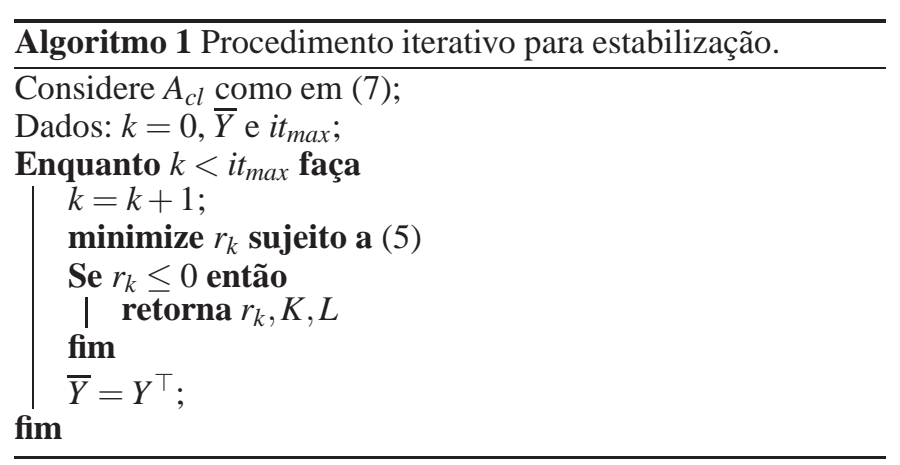

Nota-se que a sequência de valores de $r_{k}$ produzidos pelo Algoritmo 1 é não crescente. Para ver este fato, suponha que na iteração $k$ um valor finito para $r_{k}$, associado às variáveis $P=P_{k}$, $T=T_{k}$ e $Y=Y_{k}$, foi obtido. Como $\operatorname{He}(\bar{Y} Y)=\operatorname{He}\left(Y^{\top} \bar{Y}^{\top}\right)$, a escolha $\bar{Y}_{k+1}=Y_{k}$ assegura que $r_{k+1}$ não pode crescer, ou seja, $r_{k+1} \leq r_{k}$. Além disso, a convergência do Algoritmo 1 para um valor negativo de $r$ (solução estabilizante) é somente local, isto é, depende da escolha inicial $\bar{Y}=\bar{Y}_{0}$.

\section{EXEMPLOS}

Nesta seção são fornecidos exemplos que ilustram o desempenho do método proposto para realimentação de estados $\left(C_{y}=I\right)$ e de saída, comparando com os resultados obtidos em Castelan et al. (2008), incluindo o problema de controle descentralizado. A programação foi implementada no MATLAB-2015 usando o parser Yalmip (Löfberg, 2004) e o solver Mosek (Andersen and Andersen, 2000), em um computador com o sistema operacional Windows 10 core $i 7$.
Os parâmetros de entrada do algoritmo foram estabelecidos como $\bar{Y}^{\top}=\left[\begin{array}{llll}I & I & 0 & -I\end{array}\right]$, conforme sugerido no Teorema 2, e $i t_{\text {max }}=15$. Além disso, foi definida a estrutura de $Y=$ $\left[\begin{array}{llll}Y_{1} & Y_{2} & 0 & Y_{4}\end{array}\right]^{\top}, \operatorname{com} Y_{i}, i \in\{1,2,4\}$ matrizes simétricas. Considerar $Y_{3} \neq 0$ ou impor outras estruturas para as variáveis, como diagonais, arbitrárias, ou mesmo nulas, pode gerar resultados diferentes, com complexidades numéricas distintas. A escolha proposta é a que forneceu o melhor compromisso entre esforço computacional e acurácia nos exemplos apresentados a seguir.

\section{Exemplo 1}

Neste primeiro exemplo, as condições de síntese propostas são comparadas com as de Castelan et al. (2008) em termos de uma análise estatística. Foi construída uma base de dados ${ }^{1}$ de sistemas na forma (1) de acordo com os seguintes passos:

i) As matrizes $A, B_{\phi}, C_{z}$ e $D_{z}$ são criadas com elementos aleatórios (utilizando uma distribuição normal) tais que o sistema (1) satisfaça a condição de estabilidade (6);

ii) As matrizes $B_{u}, C_{y}, K$ e $L$ são geradas tais que $A-B_{u} K C_{y}$ não seja Hurwitz estável;

iii) As matrizes $A \leftarrow A-B_{\phi} K C_{y}, B_{\phi}=B_{\phi}-B_{u} L, C_{y}, C_{z}, D_{z}$, $B_{u}$ e $K$ são incluídas no banco de dados.

Considerando $n_{x} \in\{2,3,4,5\}, n_{u}=n_{z}=n_{y}=1$ e $\Omega \in$ $\{0.5,1,2.14,11.43\}$ (representando os graus 26.56, 45, 65 e 85 da abertura do setor), 100 sistemas foram gerados para cada caso (total de 1600 sistemas). A propriedade importante do banco de dados é que todos os sistemas têm a garantia de serem estabilizados pela lei de controle (4). O objetivo é comparar o Algoritmo 1 (Alg1) com Castelan et al. (2008) (Colorário 4, denominado CTQ08) na realimentação de estados e também avaliar o desempenho do método proposto no contexto de realimentação de saída.

Os resultados são mostrados na Tabela 1, que apresenta a porcentagem média dos sistemas estabilizados para cada valor de $\Omega$. A complexidade numérica é dada em termos do tempo médio ( $t$, em milissegundos) exigido para testar cada sistema e o número médio de iterações $\left(i t_{m}\right)$ demandado por Alg1.

Tabela 1. Porcentagem média de sistemas estabilizados, por $\mathrm{Alg} 1_{e}$ e CTQ08 no problema de realimentação de estados e $\operatorname{Alg} 1_{s}$ no problema de realimentação de saída, tempo médio ( $t$, em milissegundos) para testar cada sistema e número médio de iterações $\left(i t_{m}\right)$ demandado por Alg1 no Exemplo 1.

\begin{tabular}{c|ccc|ccc||cc}
\hline & \multicolumn{3}{|c|}{$\mathrm{Alg} 1_{e}$} & \multicolumn{3}{c||}{$\mathrm{Alg} 1_{s}$} & \multicolumn{2}{c||}{ CTQ08 } \\
\hline$\Omega$ & $\%$ & $i t_{m}$ & $\mathrm{t}$ & $\%$ & $i t_{m}$ & $\mathrm{t}$ & $\%$ & $\mathrm{t}$ \\
\hline 0.5 & 99 & 2.64 & 14.3 & 98.75 & 3.28 & 19.0 & 100 & 6.6 \\
1 & 98.5 & 2.49 & 13.8 & 98.5 & 3.17 & 18.6 & 100 & 4.7 \\
2.14 & 98.5 & 2.52 & 14.4 & 97.5 & 3.16 & 18.4 & 100 & 4.2 \\
11.43 & 99.25 & 2.69 & 13.5 & 97.5 & 3.32 & 20.8 & 100 & 4.4 \\
\hline
\end{tabular}

Nota-se que CTQ08 foi capaz de estabilizar todos os sistemas e $\mathrm{Alg} 1_{e}$ forneceu um desempenho ligeiramente inferior. Por outro lado, o desempenho de $\mathrm{Alg} 1_{s}$ no problema de realimentação de saída foi muito próximo ao dos resultados de realimentação de estados, mostrando a eficiência do método em um problema de difícil solução (mesmo sem considerar a não linearidade) em todas as diferentes limitações de setor.

\footnotetext{
1 A base da dados está disponível em http: //www. dt . fee.unicamp. $\mathrm{br} /$ ricfow/programs/CBA2020/LureDatabaseCBA20.zip
} 
Além disso, a elevada porcentagem de sistemas estabilizados pelo Alg1 e o número médio de iterações (sempre abaixo de 4) permitem concluir que a condição inicial escolhida para $\bar{Y}$ produziu bons resultados, embora não exista garantia de convergência em todos os casos.

\section{Exemplo 2}

Neste exemplo, o problema de controle descentralizado por realimentação de estados é investigado somente nos sistemas com $n_{x}=5$ pertencentes à base de dados do exemplo anterior. As máscaras (apenas os elementos indicados com 1 fazem parte do projeto) para o ganho $K=\left(k_{1}, k_{2}, k_{3}, k_{4}, k_{5}\right)$ são:

$$
\begin{aligned}
& K=\left[\begin{array}{lllll}
1 & 1 & 1 & 1 & 1
\end{array}\right] \quad\left(M_{1}\right), \quad K=\left[\begin{array}{lllll}
1 & 0 & 0 & 0 & 1
\end{array}\right] \quad\left(M_{4}\right), \\
& K=\left[\begin{array}{lllll}
1 & 1 & 0 & 1 & 1
\end{array}\right] \quad\left(M_{2}\right), \quad K=\left[\begin{array}{lllll}
1 & 0 & 0 & 0 & 0
\end{array}\right] \quad\left(M_{5}\right), \\
& K=\left[\begin{array}{lllll}
1 & 0 & 1 & 0 & 1
\end{array}\right] \quad\left(M_{3}\right), \quad K=\left[\begin{array}{lllll}
0 & 0 & 0 & 0 & 1
\end{array}\right] \quad\left(M_{6}\right) .
\end{aligned}
$$

A garantia de que todos os sistemas podem ser estabilizados pelas máscaras propostas pode ser obtida por meio do seguinte ajuste na matriz dinâmica do sistema

$$
A_{d c}=A+B_{u}\left(K C_{y}-K C_{y} \circ M_{i}\right),
$$

em que $M_{i}$ é a máscara desejada, $K C_{y}$ é ganho de estabilização ( $K$ disponível no banco de dados). Dessa maneira, $K_{d c}=K C_{y} \circ M_{i}$ é o ganho de estabilização descentralizado associado à máscara $M_{i}$, pois

$$
\begin{aligned}
& A_{d c}+B_{u}\left(K C_{y} \circ M_{i}\right) \\
& =A+B_{u}\left(K C_{y}-K C_{y} \circ M_{i}\right)+B_{u}\left(K C_{y} \circ M_{i}\right)=A+B_{u} K C_{y}
\end{aligned}
$$

O Algoritmo 1 é comparado com as condições de Castelan et al. (2008) (denotado CTQ08), ambos adaptados para tratar a descentralização dos ganhos. No Alg1 a adaptação é mais simples pois utiliza-se diretamente o ganho $K$ como variável de otimização, enquanto que em CTQ08 o ajuste é feito por meio de restrições em algumas variáveis de otimização, como proposto em Geromel et al. (1994). Os resultados, dados na Tabela 2, mostram uma perda de desempenho de CTQ08 quando entradas são zeradas, em relação a $M_{1}$ (estrutura completa para o ganho). Por outro lado, o Alg1 mantém a eficiência em praticamente todas as estruturas de $M_{i}$, caracterizando-se como uma abordagem menos conservadora para tratar o problema de controle descentralizado.

\section{Exemplo 3}

Considere um sistema de segunda ordem na forma (1) com matrizes dadas por

$$
\begin{gathered}
A=\left[\begin{array}{cc}
0 & 1 \\
-\omega_{n}^{2} & -2 \xi \omega_{n}
\end{array}\right], \quad B_{\phi}=\left[\begin{array}{c}
2 \\
-1
\end{array}\right], \quad B_{u}=\left[\begin{array}{c}
-1 \\
1
\end{array}\right], \\
C_{z}=\left[\begin{array}{ll}
1 & 1
\end{array}\right], \quad C_{y}=\left[\begin{array}{ll}
1 & 0.5
\end{array}\right], \quad D_{\phi}=0,
\end{gathered}
$$

$\xi=0.3, \omega_{n}=1$. A função não linear $\phi$ é composta por saturação e quantização na forma

$$
\phi=\operatorname{sat}(q(z))=\operatorname{sinal}(q(z)) \min \{\gamma,|q(z)|\},
$$

em que $\gamma>0$ é um escalar representando a amplitude máxima (simétrica) de $\phi$ e $q(z)$ é a quantização uniforme de $z$ dada por

$$
q(z)=\Delta \operatorname{sinal}(z)\left\lfloor\frac{|z|}{\Delta}\right\rfloor,
$$

\begin{tabular}{|c|c|c|c|}
\hline$\Omega$ & Máscara & Alg1 & CTQ08 \\
\hline \multirow{6}{*}{0.5} & $M_{1}$ & 100 & 100 \\
\hline & $M_{2}$ & 100 & 78 \\
\hline & $M_{3}$ & 99 & 77 \\
\hline & $M_{4}$ & 99 & 57 \\
\hline & $M_{5}$ & 99 & 60 \\
\hline & $M_{6}$ & 98 & 53 \\
\hline \multirow{6}{*}{1} & $M_{1}$ & 97 & 100 \\
\hline & $M_{2}$ & 99 & 82 \\
\hline & $M_{3}$ & 99 & 68 \\
\hline & $M_{4}$ & 97 & 58 \\
\hline & $M_{5}$ & 94 & 54 \\
\hline & $M_{6}$ & 98 & 57 \\
\hline \multirow{6}{*}{2.14} & $M_{1}$ & 97 & 100 \\
\hline & $M_{2}$ & 97 & 84 \\
\hline & $M_{3}$ & 97 & 61 \\
\hline & $M_{4}$ & 98 & 58 \\
\hline & $M_{5}$ & 96 & 53 \\
\hline & $M_{6}$ & 97 & 63 \\
\hline \multirow{6}{*}{11.43} & $\overline{M_{1}}$ & 98 & 100 \\
\hline & $M_{2}$ & 100 & 73 \\
\hline & $M_{3}$ & 99 & 59 \\
\hline & $M_{4}$ & 99 & 53 \\
\hline & $M_{5}$ & 100 & 59 \\
\hline & $M_{6}$ & 96 & 56 \\
\hline
\end{tabular}

sendo que o escalar $\Delta>0$ que determina o passo da quantização. Os valores escolhidos para este exemplo são $\gamma=3.25$ e $\Delta=0.25$ e a Fig. 3 apresenta uma ilustração gráfica de $\phi$, que é uma não linearidade limitada pelo setor definido por $\Omega=1$.
Tabela 2. Número de sistemas com $n_{x}=5$ estabilizados por Alg1 e CTQ08 para o caso de controle descentralizado utilizando as máscaras $M_{i}$, $i=1, \ldots, 6$ dadas em (10) no Exemplo 2.

Simulando o sistema com a condição inicial $x_{0}=[1,-1]^{\top}$, percebe-se que, embora a matriz $A$ seja Hurwitz, o sistema é instável, conforme mostra o gráfico superior da Fig. 4. A parte inferior da Fig. 4 mostra o valor da não linearidade ao longo da simulação, ficando claro o efeito da quantização nos primeiros 5 segundos e o efeito da saturação após 10 segundos.

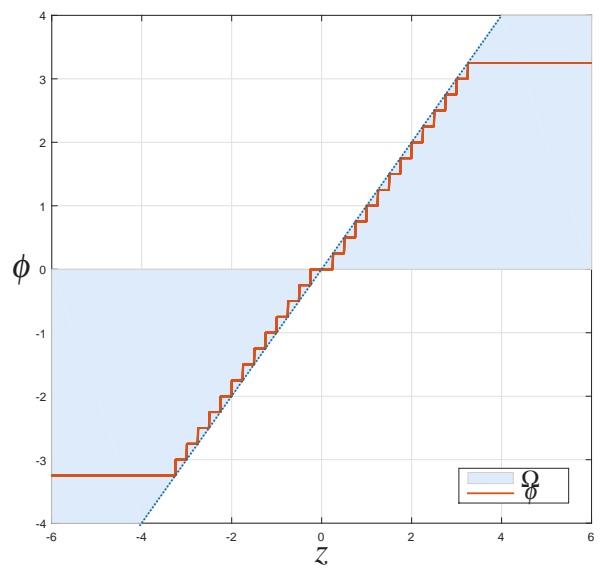

Fig. 3. Gráfico dos valores de $\phi$ (laranja) em relação a $z$ e da região delimitada pelo setor $\Omega=1$ (azul), no Exemplo 3 .

O objetivo neste exemplo é estabilizar o sistema por meio da lei de controle dada em (4), utilizando o método proposto (Alg1). Os valores dos ganhos obtidos pelo Alg1 são $K=$ 0.9994 e $L=0.8776$. Também é possível estabilizar o sistema utilizando o Alg1 sem o ganho $L$, mas nesse caso é necessário trabalhar com realimentação de estados. O comportamento do sistema estabilizado é apresentado na Fig. 5, utilizando a mesma condição inicial anterior. Como pode ser observado, os 

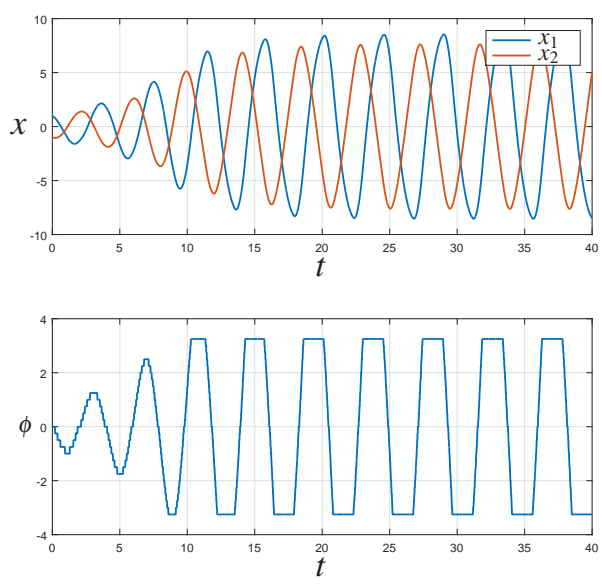

Fig. 4. Simulação do sistema apresentando o comportamento dos estados $x=\left[x_{1}, x_{2}\right]^{\top}$ e a não linearidade $\phi$, no Exemplo 3 .

ganhos são capazes de compensar o efeito da não linearidade sobre a estabilidade do sistema.
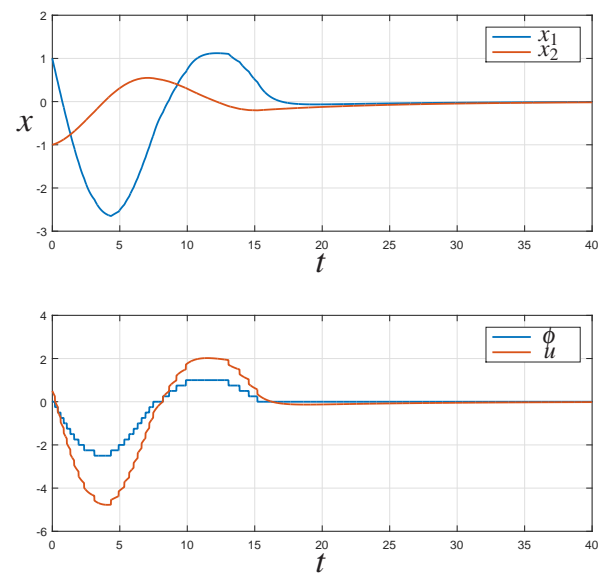

Fig. 5. Simulação do sistema estabilizado apresentando o comportamento dos estados $x=\left[x_{1}, x_{2}\right]^{\top}$, da não linearidade $\phi$ e do sinal de controle $u$, no Exemplo 3 .

\section{CONCLUSÃO}

Neste trabalho foram apresentadas condições LMIs baseadas em uma função de Lyapunov quadrática e um método iterativo para a estabilização de sistemas Lur'e contínuos no tempo com não-linearidades limitadas em setor. O controle estabilizante é feito por meio da realimentação da saída medida e da não linearidade. Os resultados equiparam-se em termos de acurácia aos fornecidos por outras condições da literatura, com vantagens no controle descentralizado, e o método pode também tratar o problema de estabilização por realimentação de saída. Como trabalhos futuros, pretende-se tratar não linearidades de setor com limitação de inclinação e também o caso discreto.

\section{REFERÊNCIAS}

Ahmad, N.S. and Carrasco, J. (2016). Convex LMI approach for stability of critically stable systems with slope-restricted nonlinearities. In Proceedings of the 55th IEEE Conference on Decision and Control, 3228-3233. Las Vegas, NV, USA.

Ahmad, N.S., Heath, W.P., and Li, G. (2013). LMI-based stability criteria for discrete-time Lur'e systems with monotonic, sector- and slope-restricted nonlinearities. IEEE Transactions on Automatic Control, 58(2), 459-465.

Andersen, E.D. and Andersen, K.D. (2000). The MOSEK interior point optimizer for linear programming: An implementation of the homogeneous algorithm. In H. Frenk, K. Roos, T. Terlaky, and S. Zhang (eds.), High Performance Optimization, volume 33 of Applied Optimization, 197-232. Springer US. http: / / www . mosek . com.

Boyd, S., El Ghaoui, L., Feron, E., and Balakrishnan, V. (1994). Linear Matrix Inequalities in System and Control Theory. SIAM Studies in Applied Mathematics, Philadelphia, PA.

Carrasco, J., Turner, M.C., and Heath, W.P. (2016). Zames-Falb multipliers for absolute stability: From O'Shea's contribution to convex searches. European Journal of Control, 28, 1-19.

Castelan, E.B., Tarbouriech, S., and Queinnec, I. (2008). Control design for a class of nonlinear continuous-time systems. Automatica, 44(8), 2034-2039.

de Oliveira, M.C. and Skelton, R.E. (2001). Stability tests for constrained linear systems. In S.O. Reza Moheimani (ed.), Perspectives in Robust Control, volume 268 of Lecture Notes in Control and Information Science, 241-257. SpringerVerlag, New York, NY.

Felipe, A., Oliveira, R.C.L.F., and Peres, P.L.D. (2016). An iterative LMI based procedure for robust stabilization of continuous-time polytopic systems. In Proceedings of the 2016 American Control Conference, 3826-3831. Boston, MA, USA.

Geromel, J.C., Bernussou, J., and Peres, P.L.D. (1994). Decentralized control through parameter space optimization. Automatica, 30(10), 1565-1578.

Gonzaga, C.A.C., Jungers, M., and Daafouz, J. (2012). Stability analysis of discrete-time Lur'e systems. Automatica, 48(9), 2277-2283.

Haddad, W.M. and Bernstein, D.S. (1993). Explicit construction of quadratic Lyapunov functions for the small gain, positivity, circle, and Popov theorems and their application to robust stability. Part I: Continuous-time theory. International Journal of Robust and Nonlinear Control, 3(4), 313-339.

Haddad, W.M. and Bernstein, D.S. (1994). Parameterdependent Lyapunov functions and the discrete-time Popov criterion for robust analysis. Automatica, 30(6), 1015-1021.

$\mathrm{Hu}$, T., Teel, A.R., and Zaccarian, L. (2006). Stability and performance for saturated systems via quadratic and nonquadratic Lyapunov functions. IEEE Transactions on Automatic Control, 51(11), 1770-1786.

Kalman, R.E. (1963). Lyapunov functions for problem of Lur'e in automatic control. Proceedings of the National Academy of Sciences of the United States of America, 49(2), 201-205.

Kapila, V. and Haddad, W.M. (1996). A multivariable extension of the Tsypkin criterion using a Lyapunov-function approach. IEEE Transactions on Automatic Control, 41(1), 149152.

Khalil, H.K. (2002). Nonlinear Systems. Prentice Hall, Upper Saddle River, NJ, 3rd edition.

Lee, S.M. and Park, J.H. (2008). Robust stabilization of discrete-time nonlinear Lur'e systems with sector and slope restricted nonlinearities. Applied Mathematics and Computation, 200, 429-436. 
Löfberg, J. (2004). YALMIP: A toolbox for modeling and optimization in MATLAB. In Proceedings of the 2004 IEEE International Symposium on Computer Aided Control Systems Design, 284-289. Taipei, Taiwan. http: / /yalmip. github.io.

Louis, J., Jungers, M., and Daafouz, J. (2015). Sufficient LMI stability conditions for Lur'e type systems governed by a control law designed on their Euler approximate model. International Journal of Control, 88(9), 1841-1850.

Montagner, V.F., Oliveira, R.C.L.F., Calliero, T.R., Borges, R.A., Peres, P.L.D., and Prieur, C. (2007). Robust absolute stability and stabilization based on homogeneous polynomially parameter-dependent Lur'e functions. In Proceedings of the 2007 American Control Conference, 6021-6026. New York, NY, USA.

Park, J., Lee, S.Y., and Park, P. (2019). A less conservative stability criterion for discrete-time Lur'e systems with sector and slope restrictions. IEEE Transactions on Automatic Control, 64(10), 4391-4395.

Park, P. (1997). A revisited Popov criterion for nonlinear Lur'e systems with sector-restrictions. International Journal of Control, 68(3), 461-469.

Tarbouriech, S., Garcia, G., Gomes da Silva Jr., J.M., and Queinnec, I. (2011). Stability and Stabilization of Linear Systems with Saturating Actuators. Springer, London, UK.

Valmorbida, G., Drummond, R., and Duncan, S.R. (2019). Regional analysis of slope-restricted Lurie systems. IEEE Transactions on Automatic Control, 64(3), 1201-1208.

Yang, C., Zhang, Q., and Zhou, L. (2007). Lur'e Lyapunov functions and absolute stability criteria for Lur'e systems with multiple nonlinearities. International Journal of Robust and Nonlinear Control, 17(9), 829-841. 